\title{
Write Permission
}

National Cancer Institute

\section{Source}

National Cancer Institute. Write Permission. NCI Thesaurus. Code C62622.

Permission that allows a user or program the ability to view information, write new information, and modify information. 